\title{
Mistuning Effects Evaluation on Turbomachine Dynamic Behaviour using Genetic Algorithms
}

\author{
Gennaro Scarselli \\ Department of Engineering for Innovation, University of Salento, Via per Monteroni, 73100 Lecce, Italy
}

Leonardo Lecce

Department of Aerospace Engineering, University of Naples “Federico II”, Via Claudio 21, 80125 Napoli, Italy

\author{
Elisa Castorini \\ Department of Engineering for Innovation, University of Salento, Via per Monteroni, 73100 Lecce, Italy
}

(Received 1 October 2010, provisionally accepted 26 July 2011, accepted 13 October 2011)

This paper presents a new approach to evaluating the mistuning effects on turbomachinery blades that is classified as neither deterministic nor statistical - it is based on the employment of genetic algorithms. A genetic algorithm has been exploited to find the structurally mistuned configuration that leads to the maximum value of blade vibration amplitude for an assigned domain of variations. A test case has been fixed and subjected to an assigned forcing field; the target of the test case was to perform a smart search of the worst mistuned configuration. The test case was a twenty-blade disc on which one thousand forced frequency response analyses have been performed. A comparison with the results, based on the Monte Carlo methods, proved the suitability and the relevance of the proposed approach. The investigation has demonstrated the applicability of this new possible engineering approach to the study of systems with uncertain properties.

\section{INTRODUCTION}

The investigation of turbine rotor dynamic behaviour usually considers all the blades that constitute the rotor as identical. Therefore, the behaviour can be described in terms of a single set of natural frequencies and mode shapes; such a disc represents a tuned system, and its vibratory properties can be evaluated by modelling only one sector of it and not the entire bladed disc. Such a circumstance - called cyclic symmetry - allows one to perform fast dynamic calculations with computational costs lower than those required when modelling the entire disc. However, the cyclic symmetry analysis is approximate because the blades are different from each other for various reasons: manufacture tolerances, material not perfectly homogeneous, deformations under load, and damages during operation. Each blade has a proper set of natural frequencies and modes different from the other blades (mistuned system). The blade vibration amplitudes and the corresponding stress levels, predicted through a forced response analysis on a symmetric model, can be completely different from the real ones leading to incorrect estimations of High Cycle Fatigue (HCF) properties of the rotor. For this reason, in the last twenty years many investigations have been carried out to evaluate the best approach for the introduction of mistuning patterns in bladed discs. ${ }^{1,2}$ The classical approaches fall into two categories: deterministic and statistical. The deterministic approach enables the modelling of the entire rotor (and not only one sector); patterns of mistuning are introduced by a perturbation of the blade geometric or physical properties from the nominal ones. ${ }^{2,3}$ Different rotor configurations are solved numerically, providing information about the influence of the assigned mistuning patterns on the rotor dynamics. Such an approach can be considered numerically exact but requires large computa- tional efforts both in terms of computational times and in terms of hardware resources allocated to obtain each solution. For this reason, in the last years, efficient numerical techniques suitable for such kinds of problems have been investigated to reduce the cost of the deterministic approach. ${ }^{4}$ Methods that utilise reduced-order modelling have been developed in order to perform a large number of computations very quickly, and these methods are currently adopted by companies in mistuning evaluations.

The statistical approach is based on the assumption that rotor structural parameters can be well described as statistical variables and solved as such: the probability that an assigned stress value occurs in one blade is provided. ${ }^{5,6}$ Such an approach does not require large computational efforts, but its reliability is strongly dependent on the consistence of the statistical model.

The random nature of real mistuning patterns coming from different manufacturing aspects makes the structural investigation of their influence a complicated topic. Four of the major concerns of the rotor industrial manufacturers are related for a given probability distribution of the blade properties: (1) the estimation of the maximum blade vibration amplitude when the rotor is subjected to the operational environments and of the corresponding stress levels; (2) the estimation of the probability that these maximum values really occur during the operational life of the engine; (3) the determination of the best and worst mistuning configuration (leading, respectively, to the minimum and maximum blade vibration amplitude) and of the corresponding response levels; (4) the evaluation of the best intentional mistuning patterns aimed to the mitigation of the effects induced by unintentional mistuning patterns.

The aim of this paper is focused on the method adopted for 